\title{
Relating the Functional Properties of an Organic Semiconductor to Molecular Structure by nc-AFM
}

\author{
By Sarah A. Burke, Jeffrey M. LeDue, Jessica M. Topple, Shawn Fostner, and \\ Peter Grütter*
}

It is well known in the field of thin-film organic electronics that film morphology plays a critical role in device properties. ${ }^{[1,2]}$ In the related field of single-molecule electronics, the local environment, that is, nanoscale structure, is crucial in determining properties such as conductance. ${ }^{[3]}$ While this has sparked significant study in the structure of molecular deposits on solid surfaces, ${ }^{[4,5]}$ it is an understanding of the connection between local structure variations and functional properties that is needed to control and optimize materials for device purposes. Moreover, despite the necessary use of insulating regions in device applications, there has been little study of structural influence on properties of molecules on insulating surfaces due to a lack of available tools. We combine the high spatial resolution of noncontact atomic force microscopy (nc-AFM) with local electrostatic characterization under laser illumination at multiple wavelengths. This allows a determination of how the observed variation in molecular-scale arrangements influences the optoelectronic properties of the prototypical organic semiconductor 3,4,9,10-perylene tetracarboxylic dianhydride (PTCDA) on a nanostructured insulator.

The electrostatic properties and surface photovoltage of various surfaces have been studied using both amplitude modulationKelvin probe force microscopy (AM-KPFM) and frequency modulation-KPFM (FM-KPFM) in ultrahigh vacuum (UHV) and in air, including both inorganic ${ }^{[6,7]}$ and organic ${ }^{[8-10]}$ model photovoltaic materials. While these studies have provided considerable insight into differences in work function and generation of charges, there has been little focus on connecting the electrostatic properties measured with local structure of the material. The use of nc-AFM in UHV allows the determination of molecular-scale structures (epitaxies) of ordered deposits, even on insulating substrates. ${ }^{[1-15]}$ Similarly, FM-KPFM and local electrostatic force spectroscopy can provide quantitatively accurate measurements of the tip-sample contact potential difference (CPD) at scales approaching the tip diameter (typically tens of $\mathrm{nm}$ ). In this work, detailed determination of the molecular-scale structure is combined with a local measurement of optical excitation using FM-KPFM and local electrostatic force spectroscopy to examine the influence of different molecular-scale structures of a model organic system on its optoelectronic properties. As this technique does not require that the

[*] Prof. P. Grütter, Dr. S. A. Burke, J. M. LeDue, J. M. Topple, S. Fostner Department of Physics, McGill University

3600 Rue University

Montreal H3A 2T8 (Canada)

E-mail: grutter@physics.mcgill.ca

DOI: 10.1002/adma.200802947 sample be conducting, in contrast to conducting AFM and scanning tunneling microscopy, even thick (bulk) insulating substrates may be used, though this complicates quantitative interpretation. The addition of illumination at multiple wavelengths allows excited molecular states to be probed, thus providing a local signature of excitation that can be connected to local structure.

The organic semiconductor PTCDA was selected due to its well-known ordered crystalline structure, ${ }^{[16]}$ easily accessible optical absorption spectrum, ${ }^{[17]}$ and the ability to trap molecular crystallites in single monolayer pits in alkali halides. ${ }^{[14,18,19]}$ Previous study of PTCDA on $\mathrm{NaCl}$ has shown a coexistence of monolayer structures with multilayer crystallites. ${ }^{[1]}$ Using nanoscale pits in $\mathrm{NaCl}$ to template the growth, these structures are observed along with confined molecular monolayers found in the pits.

The pits in the $\mathrm{NaCl}(001)$ surface, which are used to template the growth of the molecular deposit, were created by charge irradiation at elevated temperatures, and result in well-defined one-atomic-layer-deep rectangular holes in the surface. The parameters used result in pits with edge lengths of 7-15 nm, or an average area of $90-160 \mathrm{~nm}^{2}$ (some sample to sample variability), with $9 \%$ of the surface removed. Similar to what is observed for pits in $\mathrm{KBr}$, some PTCDA islands are templated by the pits, ${ }^{[14,20]}$ while some islands grow in the same manner as on the untemplated surface. ${ }^{[11]}$ This results in three types of structures: multilayer crystallites, "free" monolayer islands, and confined monolayer islands in pits. Unlike the case of PTCDA on nanotemplated $\mathrm{KBr}$, where only multilayer islands were found in pits, ${ }^{[14]}$ these were only rarely observed on $\mathrm{NaCl}$ (as in Fig. 1b), while the vast majority were filled with only a single layer.

As the coverage is increased, the dominant type of structure evolves. At 0.3 monolayer (ML), primarily monolayer structures are observed, both in the pits and on the surrounding substrate. Increasing the coverage to $0.4 \mathrm{ML}$, many more multilayer crystallites are observed, with a mixture of all three structures. Increasing further to $0.5 \mathrm{ML}$, even larger multilayer crystallites are observed, often spaced further apart, and much less of the surrounding surface is covered with monolayer islands, though confined monolayers are still quite prevalent. Compared to the untemplated growth, which exhibits a dewetting transition at 0.85 ML, the evolution from monolayer islands to multilayer crystallites appears to be shifted to lower coverage by the presence of the pits. This is consistent with the finding that highly stepped areas of the surface induce local dewetting. ${ }^{\text {11] }}$

Using the high-resolution imaging capabilities of nc-AFM, we can identify the molecular arrangement of each type of structure. The multilayer crystallites exhibit a bulk-like herringbone structure (Fig. 1e), with evidence of grain boundaries and 

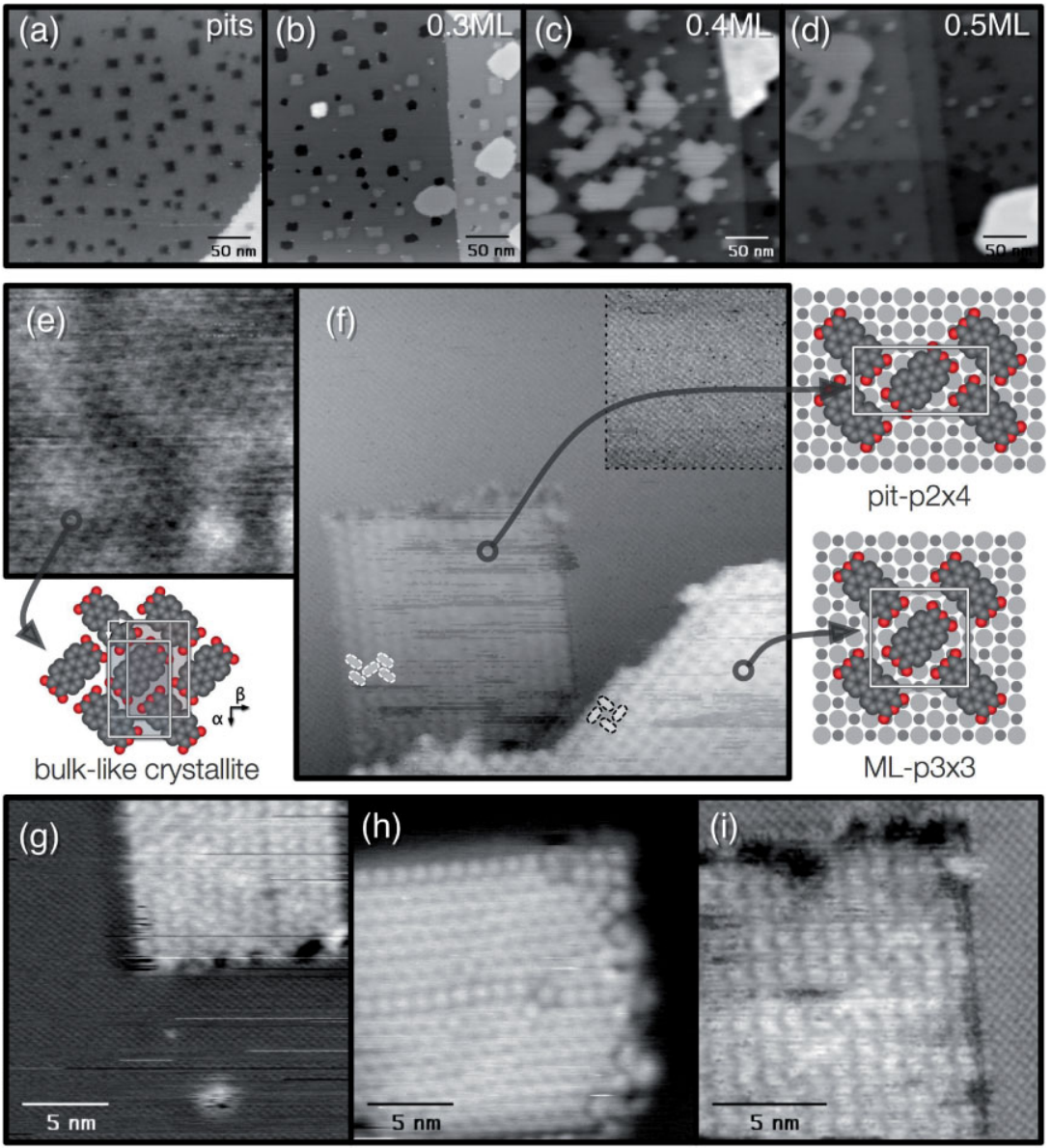

Figure 1. Top: a-d) Overview of templated PTCDA growth from pits without molecules to $0.5 \mathrm{ML}$ Three types of structures are observed for all coverages: crystallites, monolayer islands, and confined monolayer islands. Middle: Molecular scale structures with schematics of three island types, e) crystallite with bulk-like herringbone structure $(\Delta f=-11.1 \mathrm{~Hz}$, size: $15 \mathrm{~nm} \times 15 \mathrm{~nm}$, $z$-scale: $101 \mathrm{pm}), \mathrm{f}$ ) monolayer trapped in pit with $\mathrm{p} 4 \times 2$ structure, and a free monolayer with p3 $\times 3$ structure with contrast enhanced inset, to show $\mathrm{NaCl}$ lattice and dashed outlines of molecules in both structures $(\Delta f=-9.3$, size: $35 \mathrm{~nm} \times 35 \mathrm{~nm}, z$-scale: $520 \mathrm{pm})$. Bottom: $\mathrm{g}-\mathrm{i})$ examples of defects in monolayers confined in pits.

buckling of the lattice (long range periodicity). The confined and free monolayer structures have different molecular arrangements (Fig. 1f), as observed by obtaining simultaneous molecular and ionic contrast on the overlayer and substrate, respectively. The "free" monolayers have the "basketweave" p3 $\times 3$ epitaxy (measured $b_{1}=16.5 \pm 1 \AA, \quad b_{2}=17.1 \pm 0.7 \AA$, see Supporting Information for details of structure determination) observed previously for untemplated growth. ${ }^{[11]}$ The confined monolayers exhibit a herringbone-like structure with a $\mathrm{p} 4 \times 2$ registry $\left(b_{1}=21.9 \pm 0.9 \AA, b_{2}=11.2 \pm 0.6 \AA\right.$, see Supporting Information for details of structure determination), similar to $\alpha$-PTCDA. ${ }^{[16]}$ Due to the strained nature of the $\mathrm{p} 4 \times 2$ structure and the requirement imposed on the molecular crystal to fit in the confined area of the pit, a variety of defects are observed in the confined monolayers (examples shown in Fig. 1g-i).

The electrostatic properties of each of these three structures differ, as measured by KPFM and local spectroscopy (see Fig. 2). While KPFM provides a map of the local CPD even for the thick insulating substrate used, a more reliable measure of the CPD was obtained through $\Delta f$ versus $V$ spectroscopy using a parabolic fit to locate the minimum, resulting in an error on the CPD determination of $\sim 0.01 \mathrm{~V}$. Most notably, the $\mathrm{p} 3 \times 3$ monolayer structure exhibits a significantly lower CPD in the dark state compared to both the $\mathrm{NaCl}$ and the two herringbone-like structures, indicating a lower work function. The confined monolayers also show a slight negative shift of the CPD compared to the crystallites. While differences in the effective CPD can be readily measured, the applied biases are not necessarily representative of the voltage between the tip apex and the sample surface due to the capacitive voltage dropped across the large ( $3 \mathrm{~mm}$ thick) dielectric substrate used in this study. As such, care must be taken to account for the full geometry of the capacitive system involved, that is, where the biases are applied, the sample geometry and dielectric properties, and the position of the tip and cantilever beam relative to the applied biases, in order to make quantitative comparisons between measurements from different instruments. ${ }^{[21,22]}$ While great effort has been made to model the effect of tip geometry on resolution limits and accuracy of CPD measurements and KPFM, ${ }^{[23-26]}$ previous interpretation for molecular and insulating thin films assumes metallic-like behavior. ${ }^{[26,27]}$ As the dielectric properties of a thick insulating sample will clearly influence the quantitative interpretation of the CPD values here, a more complete theoretical framework for the interpretation of such data is needed. Due to the complex geometry of our apparatus, these effects are not considered, and as such only the direction and relative magnitudes of CPD shifts are considered.

Illumination of the sample provides further indication of electronic differences between the three structures. Molecules that absorb light from the laser illumination will be in an excited state, effectively lowering the work function and shifting the CPD, for this configuration, to more negative values. Three excitation wavelengths were used: 473,488 , and $514 \mathrm{~nm}$, all of which lie within the absorption spectrum of PTCDA in solution and thin films (see Fig. 3a). For all three wavelengths there is a slight upward shift of the CPD over the $\mathrm{NaCl}$ substrate, most likely a result of a small surface photovoltage excited in the Si tip. The PTCDA crystallites are the only feature that shows a consistent and significant shift for all three excitation wavelengths. Given that these have a bulk-like structure, we expect the behavior to be similar to PTCDA thin films, which show significant absorption for all three of these wavelengths.

The trapped monolayer structures show a similar trend with wavelength to the bulk-like crystallites, though much reduced in magnitude and with no significant shift with illumination at $473 \mathrm{~nm}$. The small size of the pits compared to the tip radius may 

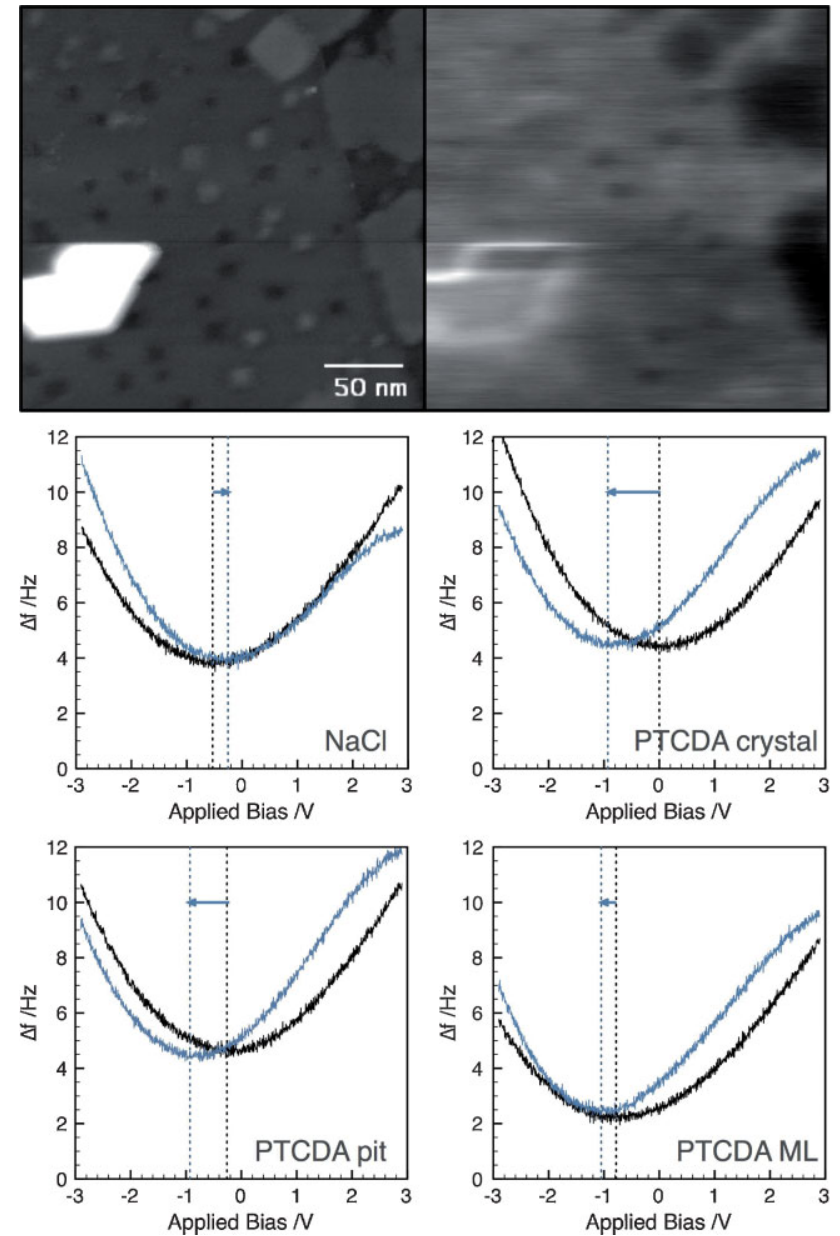

Figure 2. Topography and KPFM image of area with all three structures showing difference in CPD of each without illumination $(\Delta f=-6.9 \mathrm{~Hz}$, $\left.f_{\text {mod }}=998 \mathrm{~Hz}, V_{\text {mod }}=1.4 V_{\text {rms }}\right) . \Delta f$ vs. $V$ spectroscopy for each site shown without illumination (black) and with $488 \mathrm{~nm}$ (blue).

somewhat influence the measurement of the CPD by averaging the pit structure with the surrounding $\mathrm{NaCl}$ substrate effectively reducing the observed shift. ${ }^{[23]}$ Also, as the structure is only a single layer, electronic properties of the molecules will be more strongly influenced by the presence of the substrate, and the tightly packed nature of the structure may result in structural distortion of the molecule, influencing orbital energy levels, ${ }^{[28]}$ and shifting portions of the absorption spectrum. As the charge-transfer states are not probed at these wavelengths, the lack of such extended states in the confined structure should not affect the measurement, though the effect of confinement on these states is a subject for future study. Interestingly, the statistical variation in the CPD measured for different pit structures is significantly larger than for the other structures. The variety of defects observed in the confined monolayers (see Fig. 1g-i) are expected to influence the electronic properties, resulting in a larger variation in the measured electronic properties than for well-ordered monolayers or crystallites. Further studies correlating specific types of defects with variations in the CPD may be of interest in understanding the influence of defects on functional properties.

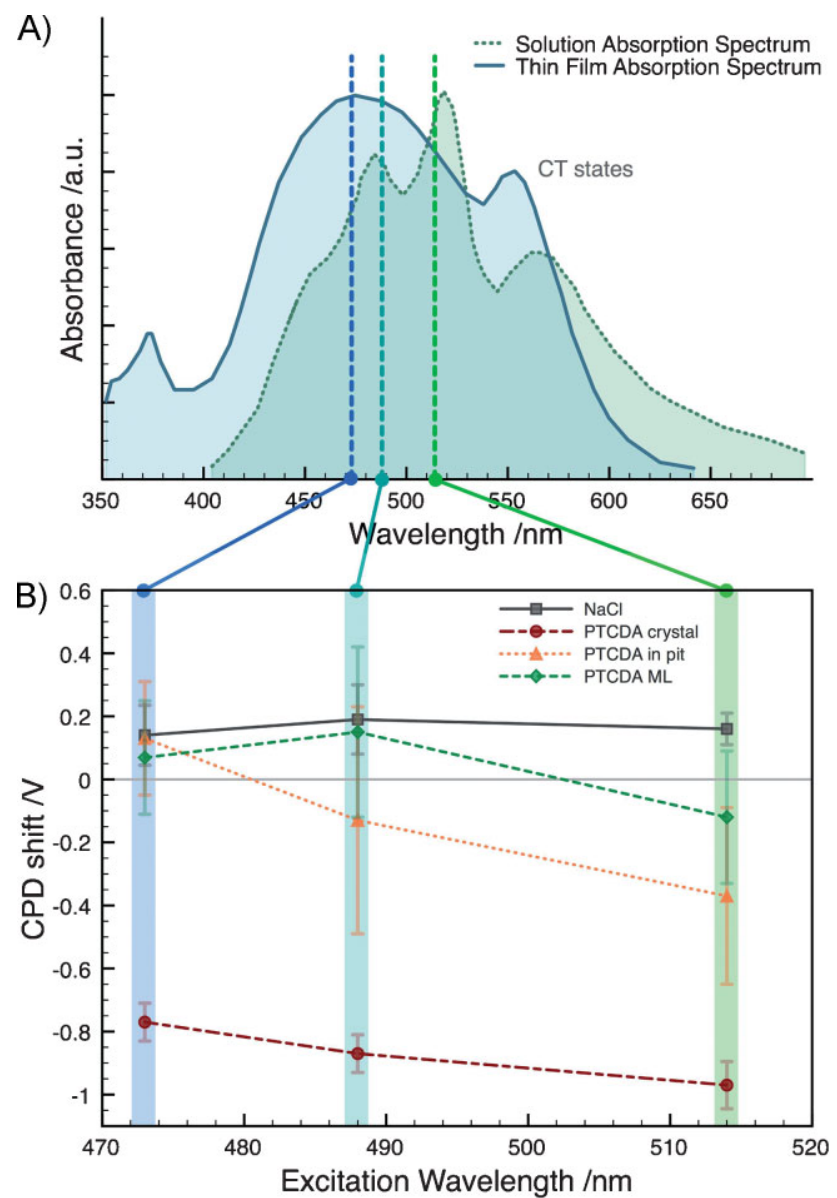

Figure 3. a) Thin-film and solution absorption spectra showing the three excitation wavelengths used [17], and b) mean photoinduced CPD shift as a function of wavelength for each site, error bars represent the standard deviation of measured CPD values at different locations, individual measurement error $\sim 0.01 \mathrm{~V}$.

The $\mathrm{p} 3 \times 3$ monolayer structure does not show any significant photoinduced shift except at $514 \mathrm{~nm}$. The extended nature of the p3 $\times 3$ structure increases the intermolecular distance and alters the arrangement of neighboring molecules with respect to each other, thus reducing the intermolecular interactions that are expected to influence molecular-orbital energies. ${ }^{[29]}$ The lack of a shift except at $514 \mathrm{~nm}$ is indicative of a monomer-like species, that is, the molecules are only weakly interacting. Schlettwein et al. ${ }^{[30]}$ observed a monomer-like signature during the early stages of PTCDA thin-film growth on $\mathrm{NaCl}$ where this structure has been observed to dominate. ${ }^{[11]}$ Control over the intermolecular spacing and intermolecular binding motif by judicious selection of substrate and the use of templating strategies could be utilized to achieve desired functional properties.

While the interpretation of the photoinduced CPD shift as representing pure electronic excitation may be oversimplified, fully understanding the represented processes requires significant modeling effort on multiple scales. The CPD shift also likely includes contributions from exciton/charge generation, and the electronic levels are expected to be modified by vibrational levels of the molecules as well as interactions with neighboring 
molecules and the substrate, as seen in a recent experimental study on a similar system. ${ }^{[31]}$ The measured CPD shift may also include a component related to polarizability, ${ }^{[32]}$ which could be altered in the excited state. Additionally, modeling of the substrate, particularly for monolayer films, need be included to understand the effects of substrate interactions, even for insulating surfaces, on electronic states. ${ }^{[28]}$ To our knowledge, no theoretical framework currently in use can account for all of these effects, though advances are being achieved in the individual areas of electron-vibrational coupling, ${ }^{[33-36]}$ substrate effects, ${ }^{[28,29]}$ and charge generation and movement in molecular crystals. ${ }^{[37]}$

In summary, nc-AFM has been used to determine the molecular arrangement of three types of structures of an organic semiconductor on a nanotemplated insulator, and locally probe the optoelectronic response of each in order to correlate structural detail with functional properties. The wavelength-dependant photoinduced shift of the measured CPD differs over each of the PTCDA structures, revealing that herringbone structures show bulk-like characteristics while extended, and therefore more weakly interacting, $\mathrm{p} 3 \times 3$ monolayers exhibit behavior more consistent with monomer-like species. By understanding the formation and influence of different molecular arrangements, the desired properties of an organic material could be tuned by simple selection of appropriate substrate or templating strategies. The successful characterization of this oft-studied optoelectronically active molecule illustrates that nc-AFM is poised to unravel the connection between nanoscale structure and electronic properties, and is applicable even to insulating surfaces, which is of critical importance to future organic-based devices.

\section{Experimental}

Single-crystal $\mathrm{NaCl}$ (Korth Kristalle $\mathrm{GmbH}$, Germany) is cleaved in situ in ultrahigh vacuum (base pressure $10^{-8} \mathrm{~Pa}$ ) to reveal (001) surfaces with atomically flat terraces of up to $1.2 \mu \mathrm{m}$. Pits are produced in the surface by exposing the sample to charge from an electron beam evaporator (operated below the deposition power for the target material) as described in Ref. [38]. The charge was generated using a tantalum target with the evaporator operated at $24 \mathrm{~W}$, below the evaporation threshold. The sample was heated to $\sim 250^{\circ} \mathrm{C}$ and exposed to the charge source for $104 \mathrm{~min}$, producing a total charge dose of $1.31 \mu \mathrm{C} \mathrm{cm}^{-2}$. This resulted in removal of $\sim 9 \%$ of the surface and pits of average area of $120 \mathrm{~nm}^{2}$ with densities of 550-1000 pits $\mu \mathrm{m}^{-2}$. PTCDA (Alfa Aesar, 98\%) molecules were deposited by thermal evaporation at $300{ }^{\circ} \mathrm{C}$ after outgassing overnight at $200-220^{\circ} \mathrm{C}$. Coverages ranging from 0.3 to $0.5 \mathrm{ML}$ at rates of $0.006-0.013 \mathrm{ML} \mathrm{s}^{-1}$ were deposited, as measured by a quartz crystal microbalance (Inficon).

The sample surface is investigated by nc-AFM, using a JEOL JSPM 4500 a, at each stage of preparation. The change in frequency of an oscillating cantilever due to tip-sample interaction is measured, and a topography is generated recording the $z$ variation required to maintain constant frequency shift [39]. A Nanosurf easyPLL is used to drive the cantilever oscillation, monitor frequency shift, and maintain constant amplitude. Highly doped Nanosensors (NCLR) Si cantilevers are used with the native oxide intact. Typical cantilevers have a resonance frequency of $170 \mathrm{kHz}$, spring constant of $40 \mathrm{~N} \mathrm{~m}^{-1}$, tip radii of $<7 \mathrm{~nm}$, and measured Q-factor in UHV of $\sim 10000$, and oscillation amplitudes of 6-7 nm were used.

Among the interaction forces detected by the nc-AFM method is the electrostatic force, which contains information about the work function of the sample through the CPD $[23,40]$. This information can be accessed by performing local spectroscopy ( $\Delta f$ vs. $V$ ), or through an imaging mode known as KPFM. In KPFM, an oscillating bias is added to a DC offset that generates a response of the frequency shift at the bias modulation frequency. This response at the modulation frequency is measured with lock-in techniques (Princton Applied Research, 5110 Lock-in amplifier), and an additional feedback circuit (JEOL SKPM control module) is employed to adjust the DC bias offset in order to null the electrostatic force, that is, the DC bias is adjusted to match the CPD. In this way, the DC bias can be recorded to generate a map of the CPD of a heterogeneous sample. Typical parameters used for this system were $f_{\text {mod }}=1 \mathrm{kHz}, A_{\text {mod }}=1-1.5 V_{\text {rms }}$, $\tau=1.0 \mathrm{~ms}, V_{\text {sens }}=50 \mathrm{mV}$ (corresponding to $\Delta f=0.9 \mathrm{~Hz}$ ), with a scan speed of $6.67 \mathrm{~ms}^{\text {point }}{ }^{-1}$.

Illumination of the sample was performed by directing laser light through the front glass port of the instrument at an angle of $\sim 10^{\circ}$. A diode-pumped solid-state laser (Melles-Griot 85-BCA-015) was used free-beam for illumination at $473 \mathrm{~nm}$ by mounting the laser directly on the air-table of the AFM. ?A3B2 tlsb=-.005w? $>$ At an output power of $7.5 \mathrm{~mW}$ and spot size of $0.6 \mathrm{~mm}$, this results in a power density of $1 \mathrm{~mW} \mathrm{~mm}$. Illumination at 488 and $514 \mathrm{~nm}$ was achieved using a tunable Argon-ion laser (Melles-Griot 35-LAP-431). Due to the size of the laser and to avoid coupling vibrations from the cooling fan to the AFM, the laser was fiber coupled, and only the fiber end with a collimator was mounted on the table. The collimated beam size was $2 \mathrm{~mm}$, with output power and power density of $26.6 \mathrm{~mW}$ or $0.4 \mathrm{~mW} \mathrm{~mm}{ }^{-2}$ at $488 \mathrm{~nm}$ and $25.1 \mathrm{~mW}$ or $0.3 \mathrm{~mW} \mathrm{~mm} \mathrm{~mm}^{-2}$ at $514 \mathrm{~nm}$. All power densities are well below bleaching thresholds, as determined from ex situ fluorescence measurements. The same polarization was used for both lasers, aligned along the sample surface (no perpendicular component).

\section{Acknowledgements}

We wish to thank J. Mativetsky and Y. Miyahara for helpful discussions regarding sample preparation and interpretation of results. We also gratefully acknowledge the following agencies for financial support: NSERC, CFI, CifAR, and FQRNT. Supporting Information is available online from Wiley InterScience or from the author. This article has been amended for print publication.

Received: October 7, 2008

Revised: February 16, 2009

Published online: April 8, 2009

[1] C. D. Dimitrakopoulos, P. R. L. Malenfant, Adv. Mater. 2002, 14, 99.

[2] N. Karl, Synth. Met. 2003, 133, 649.

[3] S. M. Lindsay, M. A. Ratner, Adv. Mater. 2007, 19, 23.

[4] G. Witte, C. Woll, J. Mater. Res. 2004, 19, 1889.

[5] F. Rosei, M. Schunack, Y. Naitoh, P. Jiang, A. Gourdon, E. Laegsgaard, I. Stensgaard, C. Joachim, F. Besenbacher, Prog. Surf. Sci. 2003, 71, 95.

[6] S. Sadewasser, T. Glatzel, S. Schuler, S. Nishiwaki, R. Kaigawa, M. C. Lux-Steiner, Thin Solid Films 2003, 437, 257.

[7] C. Loppacher, U. Zerweck, S. Teich, E. Beyreuther, T. Otto, S. Grafstrom, L. M. Eng, Nanotechnology 2005, 16, S1.

[8] H. Hoppe, T. Glatzel, M. Niggemann, A. Hinsch, M. C. Lux-Steiner, N. S. Sariciftci, Nano Lett. 2005, 5, 269.

[9] V. Palermo, M. Palma, P. Samori, Adv. Mater. 2006, 18, 145.

[10] J. Cao, J. Z. Sun, J. Hong, X. G. Yang, H. Z. Chen, M. Wang, Appl. Phys. Lett. 2003, 83, 1896.

[11] S. A. Burke, W. Ji, J. M. Mativetsky, J. M. Topple, S. Fostner, H.-J. Gao, H. Guo, P. Grutter, Phys. Rev. Lett. 2008, 100, 186104.

[12] S. A. Burke, J. M. Mativetsky, S. Fostner, P. Grutter, Phys. Rev. B 2007, 76, 035419.

[13] S. A. Burke, J. M. Mativetsky, R. Hoffmann, P. Grutter, Phys. Rev. Lett. 2005, 94, 096102

[14] J. M. Mativetsky, S. A. Burke, S. Fostner, P. Grutter, Nanotechnology 2007, 18, 105303 
[15] C. Loppacher, U. Zerweck, L. M. Eng, S. Gemming, G. Seifert, C. Olbrich, K. Morawetz, M. Schreiber, Nanotechnology 2006, 17, 1568.

[16] T. Ogawa, K. Kuwamoto, S. Isoda, T. Kobayashi, N. Karl, Acta Crystallogr. B 1999, 55, 123.

[17] S. R. Forrest, Chem. Rev. 1997, 97, 1793.

[18] L. Nony, R. Bennewitz, O. Pfeiffer, E. Gnecco, A. Baratoff, E. Meyer, T. Eguchi, A. Gourdon, C. Joachim, Nanotechnology 2004, 15, S91.

[19] J. M. Mativetsky, S. A. Burke, S. Fostner, P. Grutter, Small 2007, 3, 818.

[20] L. Nony, E. Gnecco, A. Baratoff, A. Alkauskas, R. Bennewitz, O. Pfeiffer, S. Maier, A. Wetzel, E. Meyer, C. Gerber, Nano Lett. 2004, 4, 2185.

[21] F. Bocquet, L. Nony, C. Loppacher, T. Glatzel, Phys. Rev. B 2008, 78, 035410.

[22] Private communication with L. Nony: 11th nc-AFM Int. Conf, Madrid 2008.

[23] U. Zerweck, C. Loppacher, T. Otto, S. Grafstrom, L. M. Eng, Phys. Rev. B 2005, 71, 125424.

[24] S. Sadewasser, T. Glatzel, R. Shikler, Y. Rosenwaks, M. C. Lux-Steiner, Appl. Surf. Sci. 2003, 210, 32.

[25] E. Strassburg, A. Boag, Y. Rosenwaks, Rev. Sci. Instrum. 2005, 76, 083705.

[26] U. Zerweck, C. Loppacher, T. Otto, S. Grafstrom, L. M. Eng, Nanotechnology 2007, 18, 084006.

[27] U. Zerweck, C. Loppacher, L. M. Eng, Nanotechnology 2006, 17, S107.
[28] W. Ji, S. Burke, H.-J. Gao, P. Grutter, H. Guo, submitted.

[29] J. Kroger, H. Jensen, R. Berndt, R. Rurali, N. Lorente, Chem. Phys. Lett. 2007, 438, 249

[30] D. Schlettwein, A. Back, B. Schilling, T. Fritz, N. R. Armstrong, Chem. Mater. $1998,10,601$

[3 1] T. Dienel, C. Loppacher, S. C. B. Mannsfeld, R. Forker, T. Fritz, Adv. Mater. 2008, 20, 959

[32] S. A. Burke, J. M. LeDue, Y. Miyahara, J. M. Topple, S. Fostner, P. Grütter, Nanotechnology, in press.

[33] N. Lorente, M. Persson, Phys. Rev. Lett. 2000, 85, 2997.

[34] T. Frederiksen, M. Brandbyge, N. Lorente, A. P. Jauho, Phys. Rev. Lett. 2004 93, 256601.

[35] C. T. C. Chang, J. P. Sethna, A. N. Pasupathy, J. Park, D. C. Ralph, P. L. McEuen, Phys. Rev. B 2007, 76, 045435.

[36] N. Sergueev, D. Roubtsov, H. Guo, Phys. Rev. Lett. 2005, 95, 146803.

[37] B. A. Gregg, M. C. Hanna, J. Appl. Phys. 2003, 93, 3605.

[38] J. M. Mativetsky, Y. Miyahara, S. Fostner, S. A. Burke, P. Grutter, Appl. Phys. Lett. 2006, 88, 233121.

[39] T. R. Albrecht, P. Grutter, D. Horne, D. Rugar, J. Appl. Phys. 1991, 69, 668

[40] J. M. R. Weaver, D. W. Abraham, J. Vac. Sci. Technol. B 1991, 9, 1559. 